\title{
BMJ Open Quality Training: improving antenatal detection and outcomes of congenital heart disease
}

\author{
Orhan Uzun, ${ }^{1,2}$ Julia Kennedy, ${ }^{3}$ Colin Davies, ${ }^{4}$ Anthony Goodwin, ${ }^{5}$ Nerys Thomas, ${ }^{6}$ \\ Delyth Rich, ${ }^{7}$ Andrea Thomas, ${ }^{8}$ David Tucker, ${ }^{9}$ Bryan Beattie, ${ }^{1}$ Michael J Lewis ${ }^{2}$
}

To cite: Uzun 0 , Kennedy J, Davies C, et al. Training: improving antenatal detection and outcomes of congenital heart disease. BMJ Open Quality 2018;7:e000276. doi:10.1136/ bmjoq-2017-000276

- Additional material is published online only. To view please visit the journal online (http://dx.doi.org/10.1136/ bmjoq-2017-000276).

Received 4 December 2017 Revised 7 September 2018 Accepted 16 September 2018

Check for updates

(c) Author(s) (or their employer(s)) 2018. Re-use permitted under CC BY-NC. No commercial re-use. See rights and permissions. Published by BMJ.

For numbered affiliations see end of article.

Correspondence to

Dr Orhan Uzun;

orhanuzun@hotmail.com

\section{ABSTRACT}

Objectives This study describes the design, delivery and efficacy of a regional fetal cardiac ultrasound training programme. This programme aimed to improve the antenatal detection of congenital heart disease (CHD) and its effect on fetal and postnatal outcomes.

Design setting and participants This was a prospective study that compared antenatal CHD detection rates by professionals from 13 hospitals in Wales before and after engaging in our 'skills development programme'. Existing fetal cardiac practice and perinatal outcomes were continuously audited and progressive targets were set. The work was undertaken by the Welsh Fetal Cardiovascular Network, Antenatal Screening Wales (ASW), a superintendent sonographer and a fetal cardiologist. Interventions A core professional network was established, engaging all stakeholders (including patients, health boards, specialist commissioners, ASW, ultrasonographers, radiologists, obstetricians, midwives and paediatricians). A cardiac educational lead (midwife, superintendent sonographer, radiologist, obstetrician, or a fetal medicine specialist) was established in each hospital. A new cardiac anomaly screening protocol ('outflow tract view') was created and training on the new protocol was systematically delivered at each centre. Data were prospectively collected and outcomes were continuously audited: locally by the lead fetal cardiologist; regionally by the Congenital Anomaly Register and Information Service in Wales; and nationally by the National Institute for Cardiac Outcomes and Research (NICOR) in the UK. Main outcome measures Patient satisfaction; improvements in individual sonographer skills, confidence and competency; true positive referral rate; local hospital detection rate; national detection rate of $\mathrm{CHD}$; clinical outcomes of selected cardiac abnormalities; reduction of geographical health inequality; cost efficacy.

Results High levels of patient satisfaction were demonstrated and the professional skill mix in each centre was improved. The confidence and competency of sonographers was enhanced. Each centre demonstrated a reduction in the false-positive referral rate and a significant increase in cardiac anomaly detection rate. According to the latest NICOR data, since implementing the new training programme Wales has sustained its status as UK lead for CHD detection. Health outcomes of children with CHD have improved, especially in cases of transposition of the great arteries (for which no perinatal mortality has been reported since 2008). Standardised care led to reduction of geographical health inequalities with substantial cost saving to the National Health Service due to reduced false-positive referral rates. Our successful model has been adopted by other fetal anomaly screening programmes in the UK.

Conclusions Antenatal cardiac ultrasound mass training programmes can be delivered effectively with minimal impact on finite healthcare resources. Sustainably high CHD detection rates can only be achieved by empowering the regional screening workforce through continuous investment in lifelong learning activities. These should be underpinned by high quality service standards, effective care pathways, and robust clinical governance and audit practices.

\section{INTRODUCTION}

Congenital heart disease (CHD) is the most frequent cause of congenital anomalies. When undiagnosed it has been reported that CHD is responsible for up to $10 \%$ of neonatal deaths after birth and up to half of all infant deaths. ${ }^{2}$ Antenatal detection of CHD may offer various advantages by giving an opportunity for a planned delivery, intrauterine intervention or treatment. While early detection of a cardiac anomaly allows clinicians to provide better counselling, it also gives pregnant women the opportunity to make an informed decision regarding the maintenance of pregnancy.

Prior to 2001 the antenatal detection of CHD in Wales was reported to be poor at $11 \%$ by the Welsh Congenital Anomaly Register and Information Service (CARIS). ${ }^{3}$ This detection rate of CHD in Wales was below national standards compared with other centres in the UK $(\sim 28 \%)$, according to the National Institute for Cardiac Outcomes and Research (NICOR). 4 This was partly because of variations in the ultrasound anomaly screening techniques and protocols used throughout the region. Only the four-chamber view of the fetal heart, which is known to have limited value for cardiac anomaly detection, ${ }^{5}$ was mandatory in the official anomaly screening protocol in Wales. However, it was widely accepted that the 
addition of outflow tract imaging to the four-chamber view of the fetal heart considerably improves the sonographer's ability to diagnose CHD antenatally. ${ }^{6-14}$ In light of this supporting evidence, there was a pressing need to change our national fetal anomaly screening policy and to standardise regional screening protocols.

Approximately 30000 pregnant women every year undergo fetal anomaly screening in Wales. ${ }^{3}$ Of these pregnancies around $10 \%$ would be considered to have a high risk for CHD, which in turn would require referral to a fetal cardiac specialist for detailed examination. A single fetal cardiologist covering the entire South Wales region was unable to meet this referral demand owing to limited clinic capacity. Consequently, only around 350 of the women with the restricted referral criteria which included suspected cardiac anomaly at the 20-week local anomaly scan or sustained arrhythmia could access the fetal cardiology service for further assessment, resulting in inequality in screening provision. We envisaged that dissemination of cardiac knowledge and ultrasound scanning skills to sonographers at regional centres would reduce health inequalities by facilitating access to fetal cardiac screening services in regional hospitals. This could be achieved only through teaching, practical training and skills dissemination programmes, and through better use of resources.

The Welsh Fetal Cardiovascular Network (WFCN) team, consisting of sonographers, radiologists, screening midwives, obstetricians, paediatricians with an interest in cardiology, neonatologists, fetal medicine specialists and a fetal cardiologist, in collaboration with Antenatal Screening Wales (ASW, who regulate and monitor fetal anomaly screening protocols and standards in Wales) undertook the challenge of tackling fetal cardiac health inequalities by implementing a comprehensive skills development programme on ultrasound screening of congenital heart anomalies. ASW also collaborated with Tiny Tickers in skills training programmes. Here, we report on the WFCN quality improvement project from its planning stage through to delivery. We hope that our model, with its sustained success, might help or inspire others to devise similar programmes to achieve better care for their patients too.

\section{METHODS}

This was a prospective study that compared antenatal CHD detection rates by professionals (sonographers, radiologists, obstetricians) from 13 hospitals in Wales, before and after engaging in our 'practical skills development programme'. The work was undertaken by the superintendent sonographer (NT) and fetal cardiologist (OU), both of whom were based at the University Hospital of Wales, with both representing ASW and WFCN. Collaborators included the ASW coordinator, charities, superintendent sonographers, ultrasonographer workforce, screening midwives, obstetricians and radiologists in all regional centres.

\section{Setting the objectives}

The project team aimed to deliver screening services (figure 1) that would improve patient outcomes by (1)

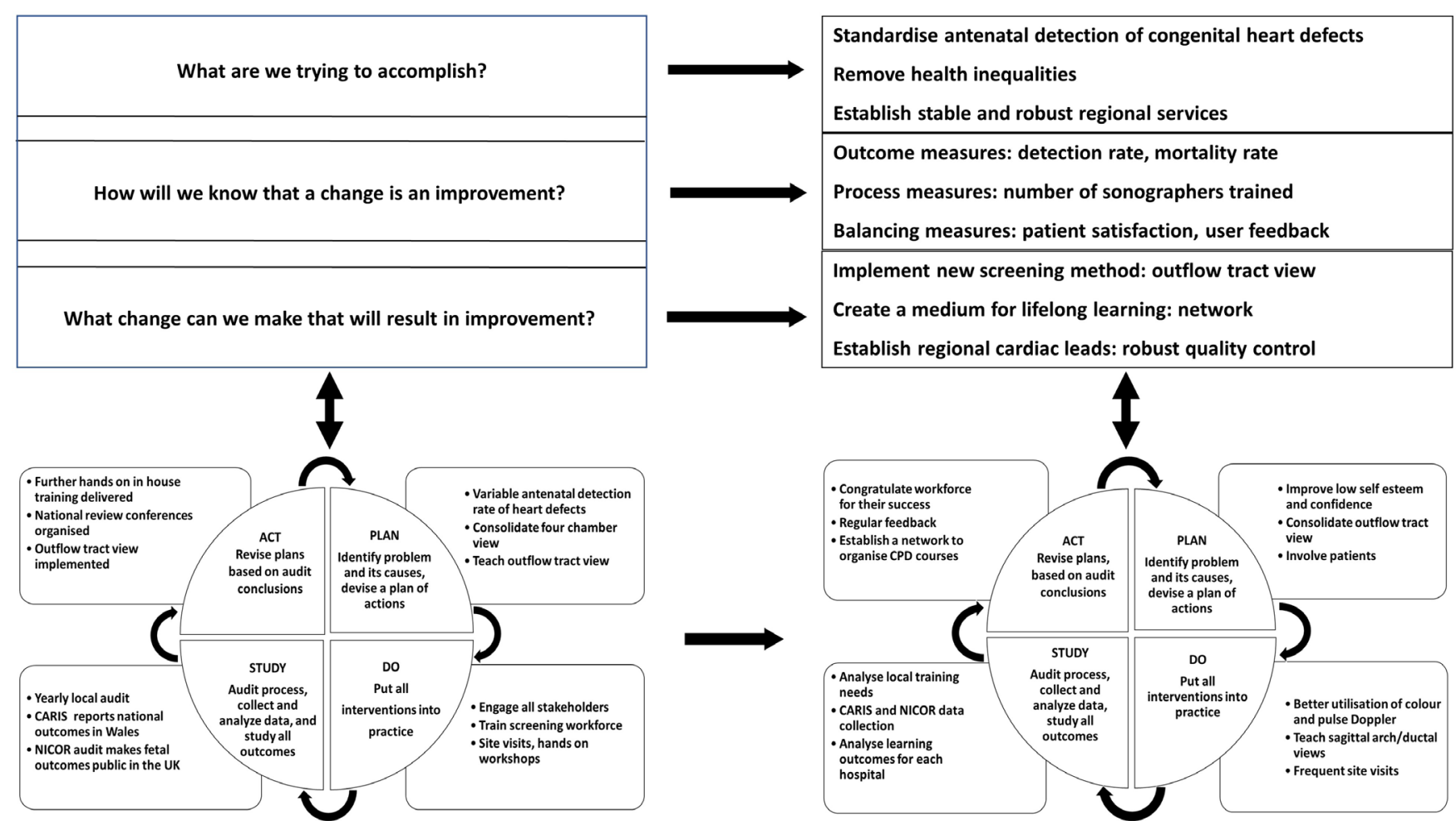

Figure 1 Plan, Do, Study, Act (PDSA) cycle for the Welsh fetal cardiac quality improvement project. CARIS, Congenital Anomaly Register and Information Service; NICOR, National Institute for Cardiac Outcomes and Research. 
Standardising regional care provision and screening protocols, including the adoption of outflow tract views as a mandatory screening protocol. (2) Using a teaching and practical skills dissemination programme to develop a workforce that is confident and competent in delivering these new protocols. The intended outcomes were (1) The provision of equitable access to fetal cardiac screening throughout South Wales. (2) Achieving more efficient use of National Health Service (NHS) resources. The timetable for achieving these goals was set at between 5 years and 7 years.

\section{Creating an inclusive team approach}

All stakeholders (including sonographers, screening midwives, radiologists, fetal medicine specialists and obstetricians) were engaged to design and deliver a series of educational lectures and hands-on training activities. This process was led by the fetal cardiologist (OU) and key sonographers (NT, JK). ASW, the Welsh Health Specialist Services Commissioner, policy makers, hospital operational managers, patients and charities were consulted in order to create a more inclusive team and thus to affect a more sustainable programme.

\section{Identifying root causes of the problem: training need} analysis, patient and service user feedback

Root cause analysis was undertaken to identify potential barriers to the confidence and competency of sonographers. An All Wales training needs analysis (TNA) was undertaken in 2007 (ASW). This provided an opportunity for sonographer feedback on confidence and competence in fetal cardiac screening at the 20-week anomaly scan, which enabled identification of training needs and requirements.

One hundred and twenty-four sonographers from 12 hospitals were asked to complete a questionnaire (see online supplementary file S1). Eighty-one responses were received. One hundred and five patients were randomly interviewed and asked to complete a questionnaire (see online supplementary file S2). Eighty fetal cardiac service users (obstetricians and midwives) were contacted and 30 of them returned feedback questionnaire forms (see online supplementary file S3). These questionnaires have been repeated at regular intervals and local and national service development has occurred as a result of these responses.

Root causes of the problem were multiple, extending from human factors to issues with machinery and software; limited leadership and the lack of a professional support network; ineffective communication channels between the fetal cardiac specialist and sonographers; absence of structured teaching and training programmes; a lack of sonographer confidence; and absence of local cardiac lead support. Figure 2 is a generic Ishikawa diagram outlining potential factors that might have a negative impact on any anomaly screening programme.

In spite of enthusiasm among sonographers for improving anomaly screening capabilities there was limited knowledge of cardiac anomaly pattern recognition among practitioners. Most importantly there was a lack of expertise in obtaining and interpreting 'outflow
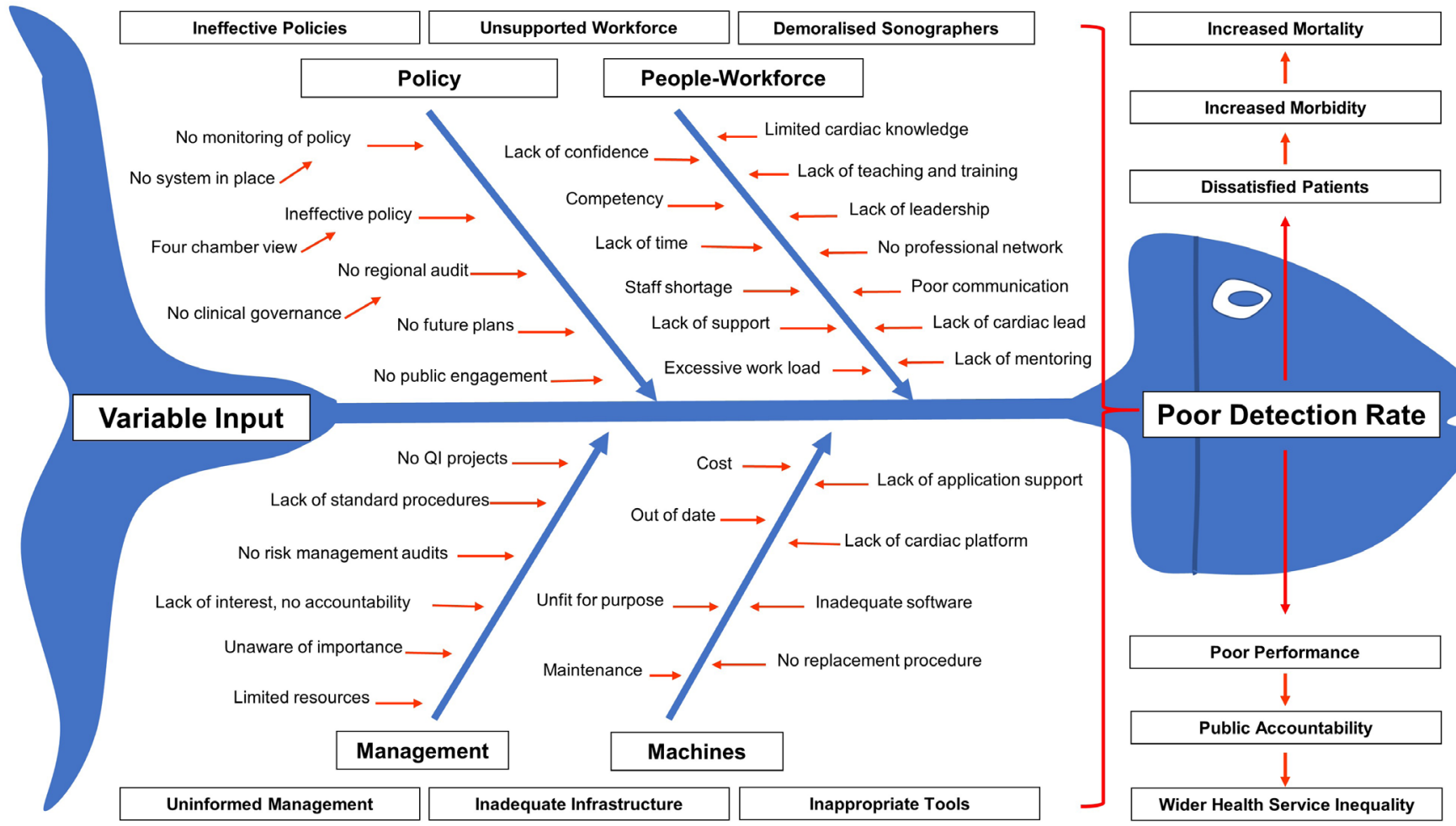

Figure 2 Ishikawa diagram showing potential causes of poor antenatal detection of congenital heart disease in any organisation. 


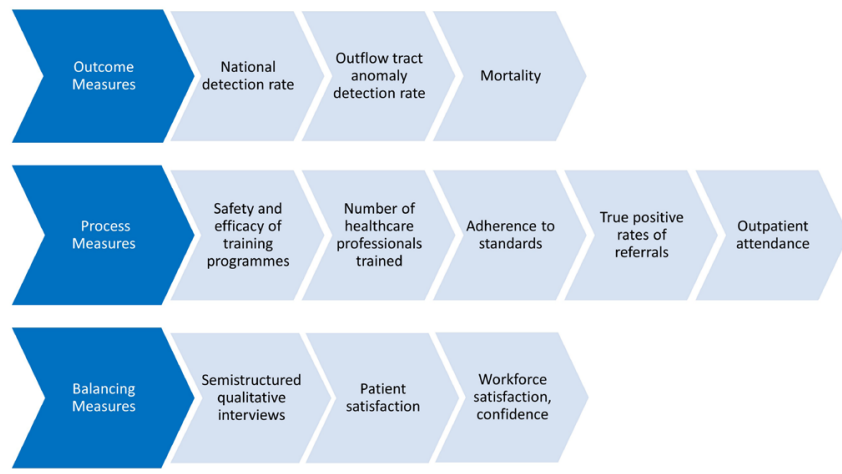

Figure 3 Project measures were determined as above.

tract view'. There was disparity in service capability and excessively variable access to specialist opinion across the local centres within South Wales. Consequently, care was not standardised. These factors had resulted in inequality and a 'postcode lottery' for specialist advise on the diagnosis and treatment of congenital heart defects in the fetus. These results were discussed and recommendations were made for an All Wales fetal cardiac training programme to be undertaken (ASW, 2007).

\section{Project measures}

A set of outcome, process and balancing measures was defined and implemented, as outlined in figure 3 . Outcome and process measures were defined with respect to improvements in individual sonographers' skills, confidence and competency; true positive referral rates for each regional centre; the national (Welsh) detection rate of CHD; and clinical outcomes of selected cardiac abnormalities.

Balancing measures were addressed via semistructured and qualitative interviews with the local centre leads, screening sonographers, screening midwives and fetal medicine obstetricians, and via patient satisfaction surveys.

\section{Team building and planning}

A series of meetings were held with stakeholders (ASW) to discuss the severity of the problem and to agree on a structured training and teaching programme to achieve equivalent standards of care in all Welsh Hospitals. A core professional network (WFCN) was established, engaging all stakeholders (including patients, health boards, specialist services commissioners, ASW, ultrasonographers, radiologists, obstetricians, midwives and paediatricians). Cardiac educational leads (midwife, superintendent sonographer, radiologist, obstetrician or a fetal medicine specialist) were then established in each hospital, specifications for a new cardiac anomaly screening protocol ('outflow tract view') were created and plans for training staff in the use of the new protocol were proposed.

\section{Patient involvement}

Patient involvement was extensive from the beginning of the process. Verbal and written patient feedback was gathered at various points of the programme. Patients were provided an opportunity to comment on all aspects of fetal cardiac screening including service delivery, counselling and communication as well as active participation in educational events.

\section{Educational strategies and interventions}

Dynamic and adaptive methods for teaching and delivering training programmes were devised to meet the needs of learners and overcome various barriers to their participation. Learning strategies included:

- Regular visits to local hospitals for theoretical knowledge enhancement and for delivering hands-on training on real patients at routine screening clinics.

- Ultrasound machine optimisation during practical hands-on training.

- Evening workshops to review prerecorded video images of the most commonly overlooked abnormalities (as identified via clinical governance) and to deliver hands-on training on real volunteer patients.

- Organisation of annual comprehensive national fetal echocardiography conferences.

- Delivery of 6-monthly network continuing professional development meetings.

- Additional structured training programmes delivered by ASW.

- The provision of performance feedback letters to screening sonographers.

- Dissemination of written guidelines, protocols and a sonographer's handbook.

- Supervised fetal cardiac scanning sessions at each cardiac centre.

- Robust clinical governance in overlooked cases.

- Regular audit and research activities.

- Facilitating sonographer's visit to fetal cardiology centre for further hands-on training.

\section{Cost implications for ongoing training}

All Wales educational interventions were funded by the Welsh Assembly Government. Other interventions were supported by programme leads as part of their professional activity and development. Trainer's time for parallel activities was facilitated by charities and educational grants from various sources.

\section{Project timeline, delivery of interventions and analyses}

A project timeline was devised for the delivery of interventions, using a structured format with continuous phase and gate analyses (figure 4). A multidimensional approach was taken to improve service provision, including preparation of a comprehensive training package based on best practice guidelines; identifying and securing charity funds for continuing education; engaging ultrasound equipment manufacturers to optimise screening equipment and thereby enabling the best possible visualisation 


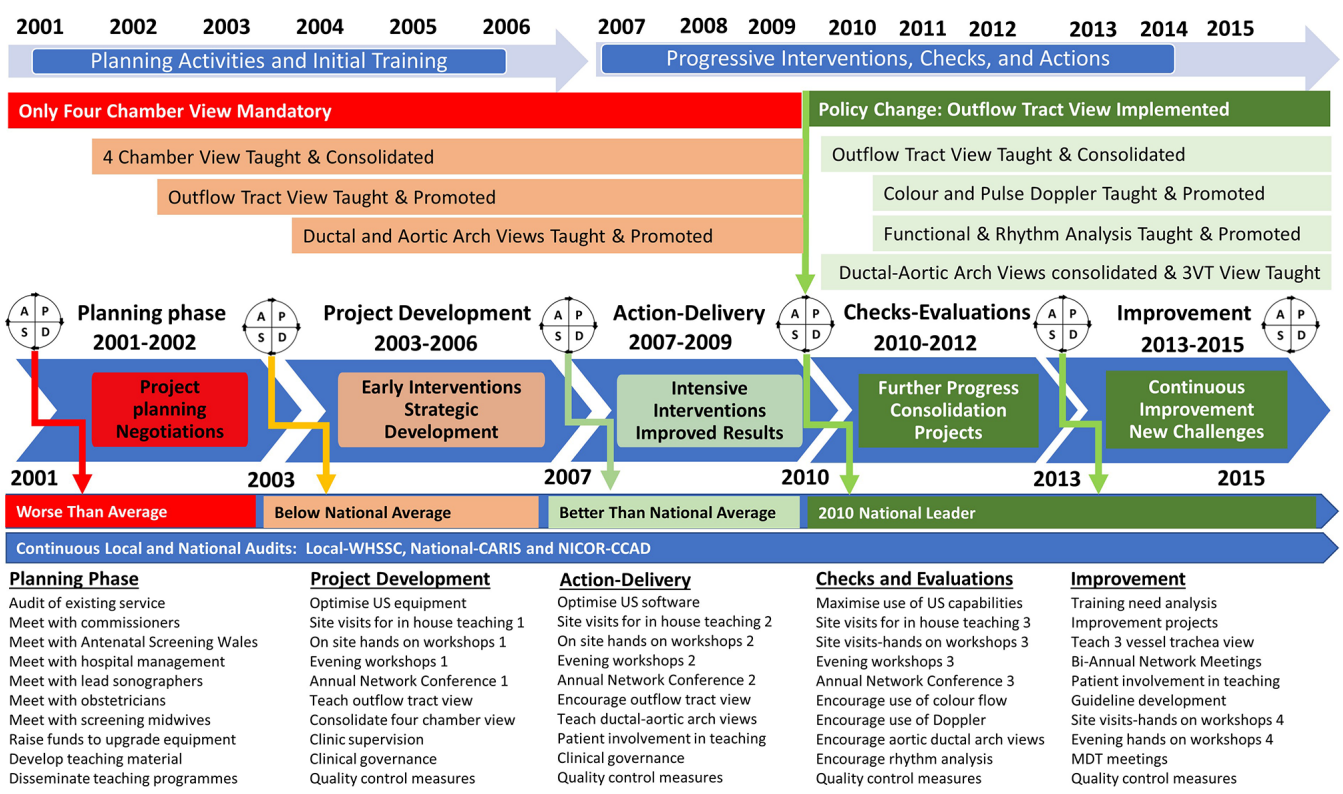

Figure 4 Project timeline and phase-gate analyses of the training programme outcomes. MDT, multidisciplinary team meetings. 3VT, three vessels and trachea.

of fetal heart structures. Together, this culminated in the proposal of new protocols and their implementation by the ASW and district general hospitals. ASW commissioned a parallel training programme with a charity called Tiny Tickers.

\section{Engaging policy makers to change screening protocols}

Prior to 2010 only the four-chamber heart view was a mandatory component of the 20-week anomaly screening protocol in Wales; this view affords a reported antenatal identification of only $23 \%$ of cardiac abnormalities in the fetus. ${ }^{10}$ However, it has been widely accepted for many years that the addition of the 'outflow tract view' increases this rate substantially. ${ }^{9-14}$ Therefore there was a pressing need to change the existing anomaly screening policy by implementing the additional outflow tract view, with the intention of increasing the detection rates of heart defects and subsequently improving health outcomes.

\section{Access to training opportunities}

All institutions delivering the 20-week fetal anomaly screening were offered training days which were organised at the convenience of both the department and the trainer. The time spent in each institution was variable and largely dependent on the number of staff undertaking fetal anomaly screening. Individual institutions organised the staff that would attend the training sessions and a register of attendees was kept by both the institution and the trainer. During training days, each staff member had an opportunity to spend 1 day observing and scanning with the trainer. Interactive training was undertaken on the basis of a minimum number of examinations, so time was variable, although all staff undertook at least three detailed examinations with the trainer. There were inevitable instances of staff sickness or leave so if no subsequent training day was available at the institution, those that were absent were invited to attend a specialist fetal echocardiography clinic or, where possible, repeat sessions were organised.

\section{Accountability, clinical governance, training need and outcomes}

The lead fetal cardiologist, cardiac lead sonographers, superintendent sonographers, ASW and the screening workforce assumed shared accountability. Data were prospectively collected and outcomes were continuously analysed locally by the lead fetal cardiologist. The educational needs and practical skills of sonographers were evaluated by conducting repeated training-need analyses. Antenatal detection rates of CHD, centre-specific referral patterns, and perinatal mortality rates for selected cardiac anomalies were determined locally by annual audit, nationally by Congenital Anomaly Register and Information Service (CARIS) in Wales ${ }^{3}$ and by National Institute for Cardiac Outcomes and Research (NICOR) ${ }^{4}$ in the UK. The implemented protocol changes were reinforced by active participation of the stakeholders. This was achieved via ongoing training and by dissemination of audit results and practice analyses.

\section{RESULTS}

\section{Effects of changes}

Policy changes, detection rates and sonographer confidence

The All Wales Training Programme completion was accomplished by 2009, as envisaged in the phase-gate quality improvement model (figure 4). In September 2010, ASW implemented the mandatory examination of outflow tracts at 20-week anomaly screening protocol in compliance with professional guidelines. ${ }^{6-8} 15$ This served to standardise care and reduced the hitherto 
A

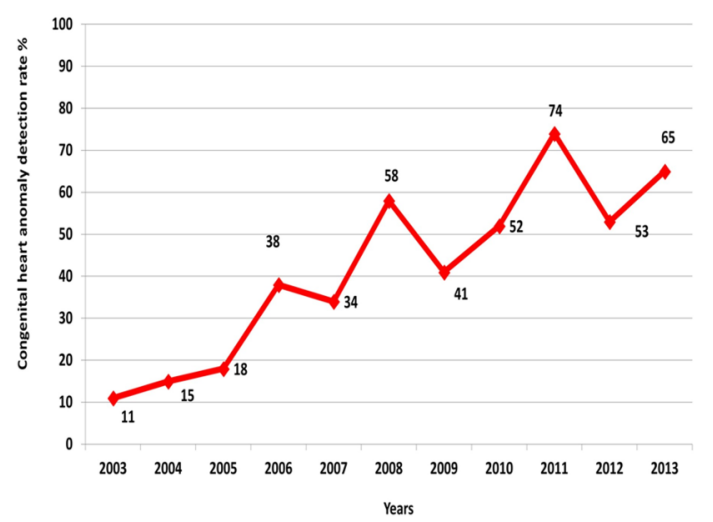

B

\begin{tabular}{|l|c|c|c|c|c|c|}
\hline Country & $\mathbf{2 0 1 0 - 2 0 1 1}$ & $\mathbf{2 0 1 1 - 2 0 1 2}$ & $\mathbf{2 0 1 2 - 2 0 1 3}$ & $\mathbf{2 0 1 3 - 2 0 1 4}$ & $\mathbf{2 0 1 4 - 2 0 1 5}$ & $\mathbf{2 0 1 5 - 2 0 1 6}$ \\
\hline England & $38 \%$ & $40 \%$ & $43 \%$ & $47 \%$ & $47 \%$ & $50 \%$ \\
\hline Ireland (Rol) & $22 \%$ & $37 \%$ & $33 \%$ & $38 \%$ & $49 \%$ & $54 \%$ \\
\hline Northern Ireland & $32 \%$ & $36 \%$ & $34 \%$ & $39 \%$ & $50 \%$ & $47 \%$ \\
\hline & & & & & & \\
\hline Scotland & $30 \%$ & $37 \%$ & $47 \%$ & $38 \%$ & $45 \%$ & $43 \%$ \\
\hline Wales & $47 \%$ & $61 \%$ & $56 \%$ & $55 \%$ & $49 \%$ & $59 \%$ \\
\hline & & & & & & \\
UK and Rol together & $36 \%$ & $40 \%$ & $42 \%$ & $46 \%$ & $47 \%$ & $50 \%$ \\
\hline
\end{tabular}

Figure 5 (A) Trend in detection rate of all congenital heart diseases in Wales (Congenital Anomaly Register and Information Service). (B) Comparison of antenatal detection rate of significant congenital heart diseases requiring operation within the first year of life in the UK (NICOR-CCAD 2010-2016 data). Wales became the lead nation in 2009 and has sustained its status to date. NICOR, National Institute for Cardiac Outcomes and Research.

postcode inequality in Wales with regard to the detection of fetal heart defects.

The impact of training was noted very quickly and after the first annual audit a generally increasing trend of antenatal anomaly detection was demonstrated. CARIS and NICOR data confirmed that the overall detection rate of CHD increased from $11.3 \%$ in 2003 to $37.8 \%$ by 2006 (figure 5A). This increase in detection rate was sustained and led to Wales becoming the lead nation for CHD detection in the UK by 2010 , with a detection rate of $52 \%$ (figure 5B). A further substantial increase in detection was noted following the All Wales Training Programme and the mandatory implementation of outflow tract examination in 2010/2011, when overall detection rates rose from $52 \%$ to $73.5 \%$. However, despite remaining well above the national average, there was a subsequent decline in the overall detection rate to $65 \%$ in 2013 (figure 5A).

This project improved the overall detection rates of four-chamber and outflow tract anomalies (figure 6), it also significantly impacted on the specific detection rates of major fetal heart defects (figure 7) and it reduced

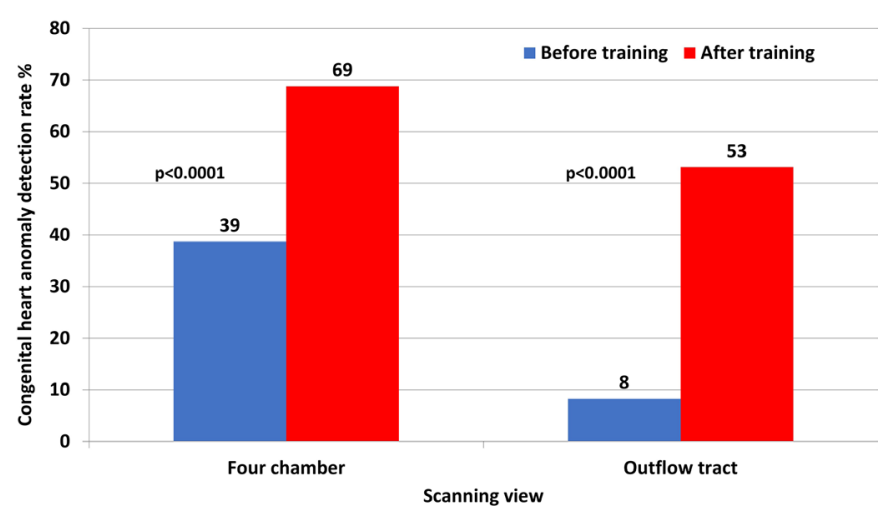

Figure 6 Improved overall detection rates of 'four chamber' and 'outflow tract' congenital heart disease anomalies after training (Antenatal Screening Wales: http://www. antenatalscreening.wales.nhs.uk/). false-positive detections from $85.7 \%$ to $25.8 \%$ throughout the study (see figure 8 for true-positive detection rates). ${ }^{34}$

\section{Training requirements for sonographers}

Even those sonographers who stated that they were very confident performing the four-chamber and outflow tract views expressed a need for ongoing training. Although the need for training on the four-chamber view was less obvious, there was a substantive requirement for training in examination of the outflow tracts.

When compared with the 2008 sonographer TNA, the 2015 TNA suggested that sonographer confidence in examining both the four-chamber and outflow tract views had increased to $87 \%$ and $61 \%$, respectively, in 2015 , compared with $70 \%$ and $18 \%$ in 2008 (figure 9). However, there was still significantly lower confidence among sonographers in examining outflow tracts compared with the four-chamber assessment, with $87 \%$ of sonographers expressing a need for ongoing training in this procedure.

\section{Improved patient outcomes}

Perinatal morbidity and mortality (death within 7 days of birth) non-chromosomal congenital heart defects in Wales was substantially reduced ((figure 10). This was as a result of the antenatal detection rate increasing from below $10 \%$ to above the national (UK) average for all congenital heart anomalies, and to over $90 \%$ for the life-threatening heart defects associated with hypoplastic left and right heart (CARIS 2012). Prior to 2008 there was a $10 \%$ preoperative mortality in babies with transposition of the great arteries (TGA), but mortality has been reduced to $0 \%$ (death within 30 days after surgery) since the introduction of the new imaging protocols (figure 11) and the survival of babies with HLHS has increased from $30 \%$ to over $75 \%$ (30 days survival after surgery).

\section{Staff and patient feedback}

Positive medical staff and patient feedback was obtained by named cardiac screening leads and screening midwives 


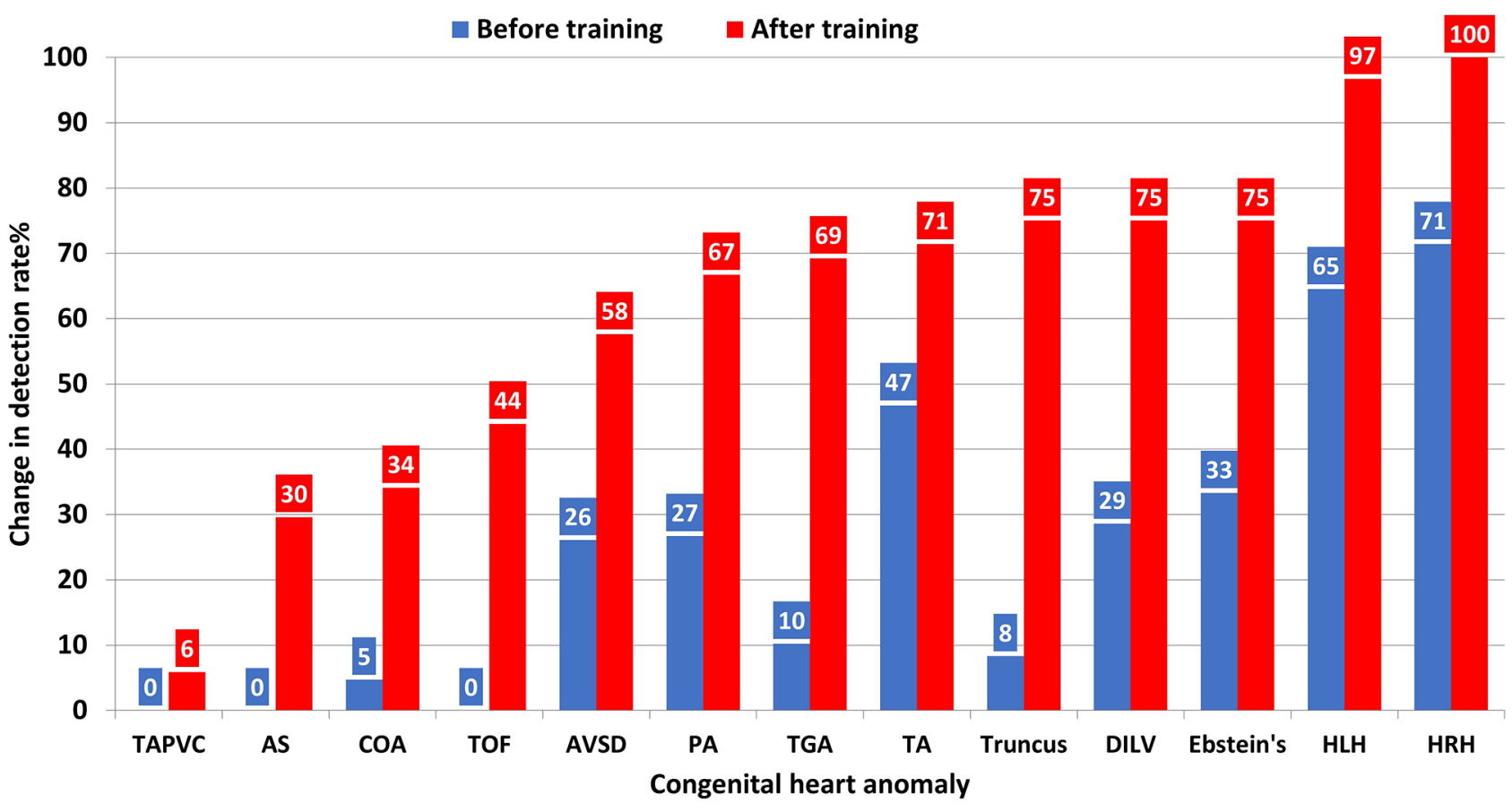

Figure 7 Change in detection rates of specific congenital heart defects (NICOR-CCAD). NICOR, National InstituteforCardiacOutcomesand Research. CCAD, Congenital Cardiac Anomaly Register. TGA, transposition of the great arteries. TAPVC, total anomalous pulmonary venous connection. AS, aortic stenosis. COA, coarctation of the aorta. TOF, tetralogy of Fallot. AVSD, atrioventricular septal defect. PA, pulmonary atresia. TA, truncus arteriosus. DILV, double inlet left ventricle. $\mathrm{HLH}$, hypoplastic left heart. HRH, hypoplastic right heart.

after each training or teaching activity. Medical staff statements included: 'our confidence increased substantially', 'better informed about cardiac defects', 'feel supported', 'became more enthusiastic about new screening view', 'network meetings invaluable'. Patients feedback statements included: 'felt listened (to)', 'felt supported', 'fantastic team work', 'felt valued as a parent to participate in network teaching activities', 'very personal care'.

Patient suggestions included the following items: (1) Improve waiting time for scans. (2) Actual consultation

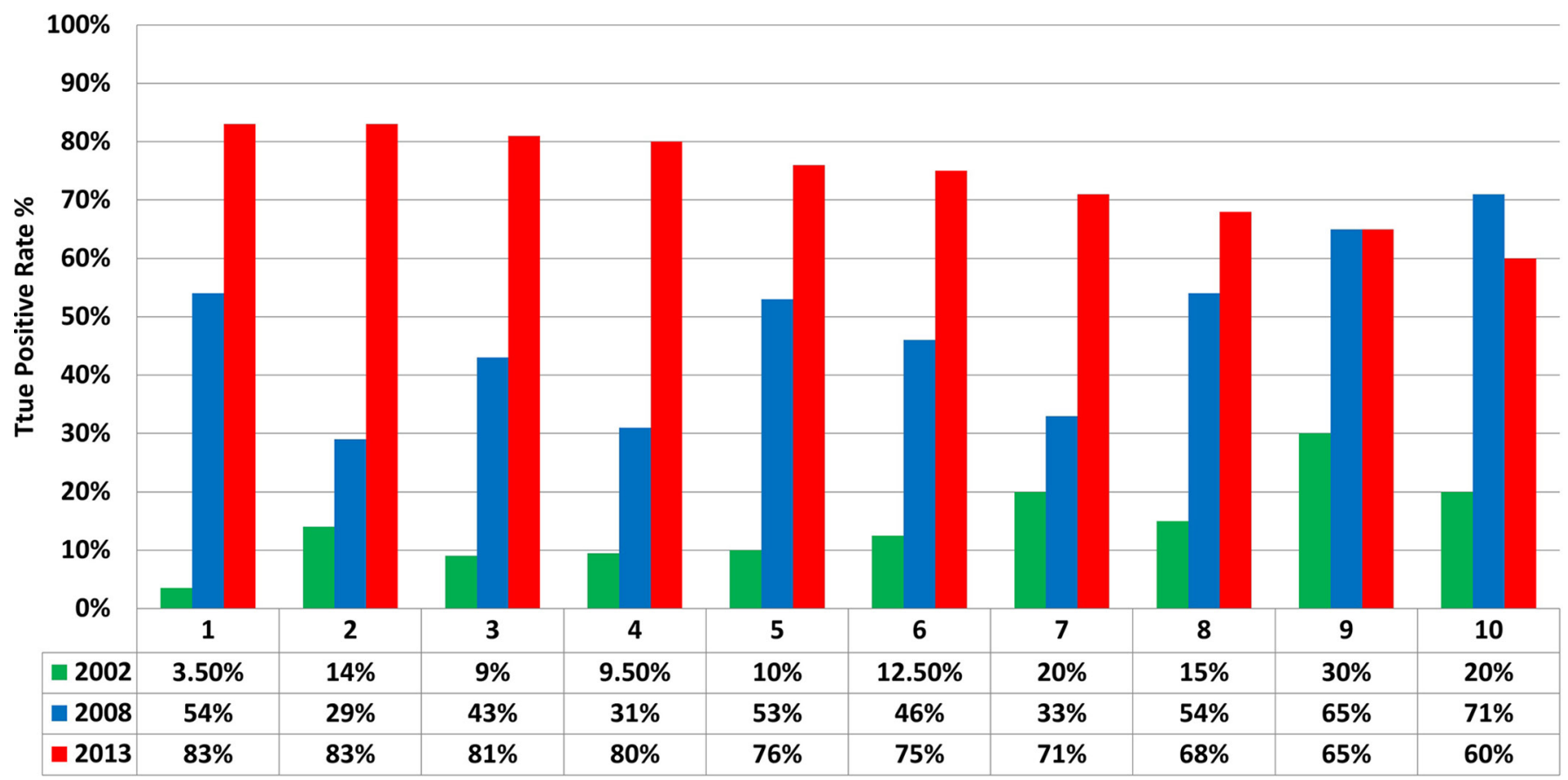

Referring Center

Figure 8 Change in true-positive rates among patients referred for specialist cardiac scan (NICOR-CCAD). NICOR, National Institute for Cardiac Outcomes and Research. 


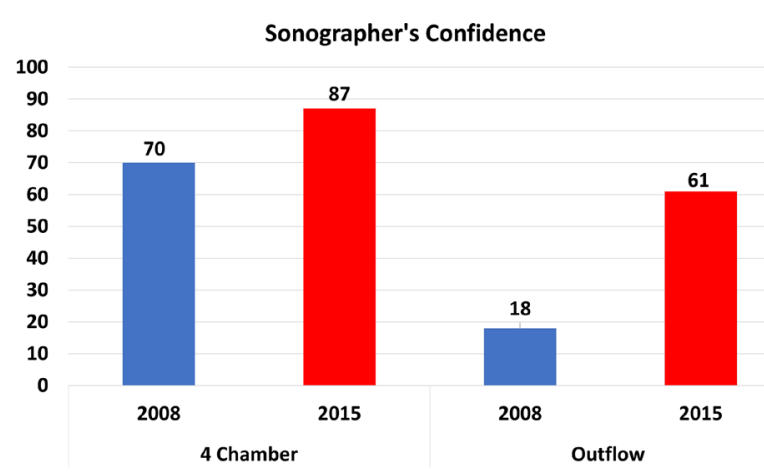

Figure 9 Change in sonographers' confidence in obtaining four-chamber and outflow tract views before and after training in 2008 and 2015 (Antenatal Screening Wales).

letters to be sent to them. (3) Dedicated communication process. Sonographer feedback was obtained at regular intervals by means of local and national forums. In addition, individual sonographer and departmental feedback was provided on a specific case basis. Sonographers' suggestions comprised the following: (1) More hands-on training to be delivered in the evenings at the specialist centre by the fetal cardiologist (2) Sonographers' visits to be facilitated to see anomalies and attend specialist clinics. (3) Sonographers' handbook and educational material to be disseminated. Appropriate responses and actions to the above suggestions were formulated and delivered to individuals or regional teams.

\section{Improved clinical efficiency and more effective reporting of results}

Improved provision of local care and the subsequent reduction in the number of tertiary referrals resulted in the availability of spare outpatient slots each week. This allowed the fetal cardiologist to see urgent cases more quickly and to dedicate more time to a larger number of patients with positive diagnoses of CHD. In addition, reduced pressure on outpatient clinic space enabled fetal cardiologists to have more time for interaction and more effective communication pathways between fetal cardiac liaison nurses, perinatologists, referring obstetricians, sonographers and regional

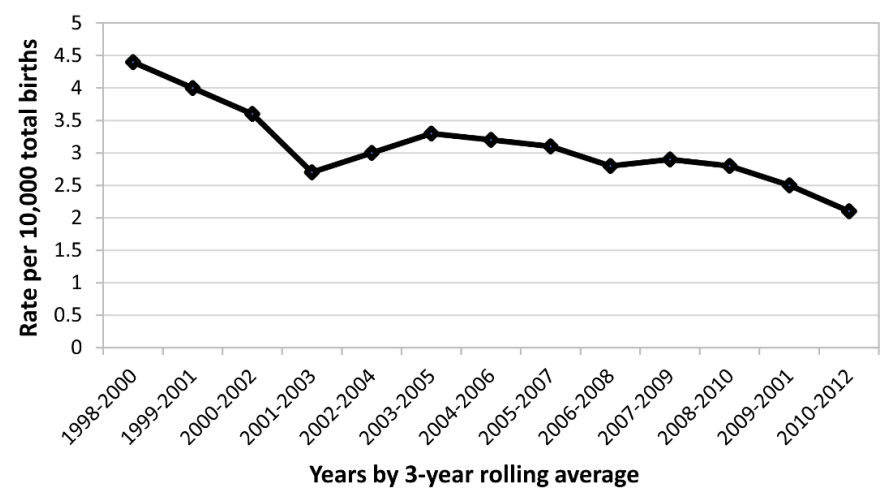

Figure 10 Three-year rolling average of perinatal mortality (death within 7 days of birth) in non-chromosomal congenital heart disease in Wales during 1998-2012 (Congenital Anomaly Register and Information Service).

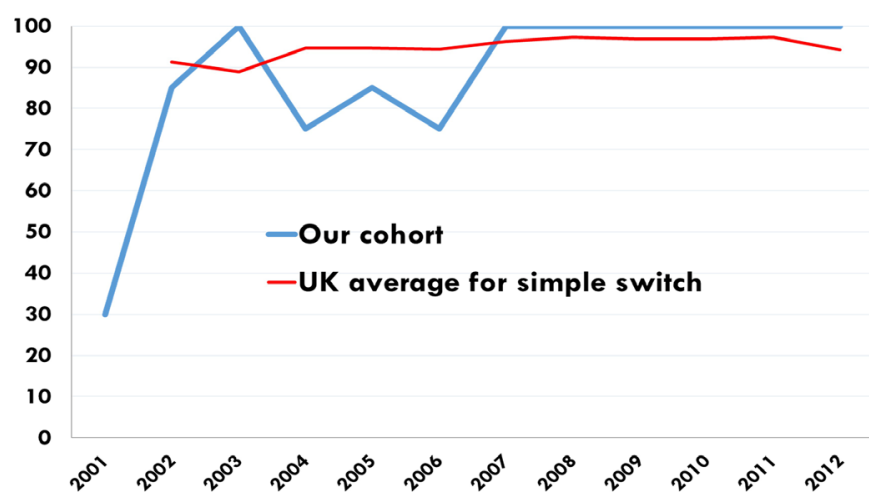

Figure 11 Impact of improved antenatal detection rate on the outcome of arterial switch operation for transposition of the great arteries. Comparison of 30-day postoperative mortality between our cohort and UK cumulative data according to the National Institute for Cardiovascular Outcomes Research and the National Congenital Heart Disease Audit websites.

cardiac leads. Fetal cardiac liaison nurses and cardiac lead sonographers in each peripheral hospital were able to establish more effective reporting channels by direct interaction with scan results, and thereby to make recommendations for intervention in a timelier fashion.

\section{Cost saving}

The total cost saving to the NHS and society was estimated to be $£ 227500$ per year. This calculation was based on the resource implications of the reduction of false-positive referrals, unnecessary fetal echocardiography examinations, avoidance of the loss of parental income, and avoidance of the costs of travel and CPD activities.

The number of referrals for fetal echocardiography was reduced from 550 to 300 per year between 2004 and 2014. With a cost of approximately $£ 400$ per referral this provided a service saving of $£ 100000$ for the NHS, in addition to travel and time cost savings of $£ 12500$ per year for patients.

The majority of fetuses with severe CHDs were diagnosed antenatally and were delivered at a surgical centre. This obviated the need for urgent transfers of sick babies to a designated surgical centre, as would be necessary with postnatally detected severe heart defects. This resulted in a cost saving of approximately $£ 100000$ per year based on an average of 30 extra sick neonates requiring emergency transfer each year (one emergency transfer costing approximately £3500). Establishment of WFCN provided a free educational platform for ongoing training and CPD in fetal echocardiography for staff. With approximately 60 staff attending two full study days annually and based on the average 2-day fetal echo course fee of $£ 250$ per person, this equates to a saving of $£ 15000$ per year.

Trainer's time for parallel teaching activities was cost-neutral due to contributions from charities and non-restricted educational grants from various sources. 


\section{DISCUSSION}

\section{Achievements of the Welsh fetal cardiac screening} programme

The Welsh quality improvement project has led to better antenatal detection rates of fetal heart anomalies, the standardisation of care provision and improved patient outcomes through an extensive training programme of skill dissemination and service transformation in peripheral hospitals. Implementation of a new screening method, the outflow tract view, helped Wales move from its previously below-national-average position to having the leading level of antenatal heart anomaly detection in the $\mathrm{UK}^{34}$

Previous studies have shown that cardiac anomaly detection rates can be improved if appropriate protocols are used and the screening workforce is supported with adequate resource allocation for continuous education and training. ${ }^{3}{ }^{46-8}$ The significant increase in antenatal detection demonstrated in our project was achieved through the implementation of both amended protocols and mandatory training, and with minimum financial impact on the NHS. Our quality improvement project was taken as an exemplar for the Fetal Anomaly Screening Programme (FASP) in England and it provided an inspiration for similar projects in the UK.

However, our regional and NICOR audit results did show a temporary reduction in CHD detection rates in 2013-2015, during which time the provision of training was more challenging owing to staffing shortages and reduced funding provision for continuing education and training. ${ }^{4}$ As this was subsequent to the mandatory implementation of the outflow tract protocol, it has been proved that while protocol development and implementation has a substantial impact on detection rates, sustainability of standards can only be achieved by a parallel and continual investment in training.

\section{Improved patient outcomes}

The design and delivery of this programme of service transformation, reinforced by comprehensive data collection and prospective evaluation of the entire process, has demonstrated clear improvements in fetal cardiac service delivery and patient outcomes in Wales. Increased antenatal detection of CHD may result in better patient outcomes and improved surgical results. ${ }^{16-19}$ However, some studies have failed to demonstrate any survival benefit despite increased antenatal detection rates in selected cardiac anomalies. ${ }^{20}$ On the contrary, the present study has changed the health map of a nation in terms of screening efficacy, and it has led to a substantial reduction in perinatal morbidity and mortality (within the first 30 days) from heart defects. The difference between our results and the abovementioned study ${ }^{20}$ can be attributed to the higher proportion of life-threatening cardiac abnormalities being detected antenatally in our study. In particular, patients with antenatal transposition of the great arteries (TGA) had the highest survival improvement as a result of better antenatal detection, in keeping with a previous study. ${ }^{18}$ Elective planned delivery of these babies resulted in optimum presurgical conditions of the newborns which in turn translated into better postoperative outcomes.

\section{Training processes}

The training process involved a steep learning curve for professionals across each of the centres in South Wales, involving numerous local training meetings and collaborative workshops. The lead fetal cardiologist continued to visit each centre to undertake regular training; this provided the advantage of training in the local screening environment and using the equipment with which the sonographers were familiar. Regular one-to-one hands-on training (by sitting behind sonographers as they worked) facilitated demonstration of the ultrasound transducer manipulation that is required to obtain optimum views of the fetal heart. Regular local audits were held to measure progress of the individual centres by the cardiac leads, which also helped modify project targets when any shortcoming was identified. This quality control process was conducted in the most supportive manner possible, rather than being seen as a threat to the individual sonographer's working practice.

What is unique to this study is that multidimensional teaching-training tools were used to deliver educational objectives which were effectively administered via the 'network' approach. The training process was proposed as a new learning opportunity and it was delivered over a period of time rather than being enforced as compulsory implementation of a policy with a strict timeline. As a result, before the outflow tract screening policy was implemented, most centres were already prepared to undertake new challenges with minimum additional training. This study has shown that the parallel and coordinated work of policy makers and professional networks is a model of how public health policies can be delivered successfully in the most cost-effective way.

\section{Cost implications}

A cost saving has been identified for both the health service and the patient, and this was further enhanced by an improvement in the patient experience, especially for those parents living remotely from the referral centre. Some patients were having to travel for over 4 hours for an appointment while others were waiting up to 4 weeks for a specialist opinion. A substantial cost saving to both NHS and public was demonstrated when considering the reduced burden of unnecessary referrals, the reduction of urgent transfer of sick neonates, loss of work time and travel costs.

Sustainability of service delivery has been achieved by adopting the changes in fetal cardiac scanning and ongoing training into the well established All Wales antenatal screening programme. This was accomplished by identifying cardiac leads within each locality and developing local expertise. 


\section{Improved patient satisfaction}

The increase in sonographer confidence and competence, along with the provision of a cardiac lead, allowed for prompt treatment to be started locally using standardised guidelines. This enhanced local staff expertise and confidence and promoted a more family centred process for the patient. The more localised provision of care minimised the disruption to family life of parents, limited their loss of work and possibly their earnings, and elicited extremely positive feedback from the parents on their experience of the service. Patient involvement in the training and service design was another accomplishment of this study, with patients feeling valued by being given a pivotal role in all educational and quality improvement activities rather than being seen as passive participants or serving as simple fundraisers.

\section{Restricted referral criteria}

As a result of excess referrals to one fetal cardiologist with only one funded clinic per week there was inadequate capacity to see the number of patients referred. As a consequence, referral criteria were restricted to either a suspected cardiac anomaly on the 20-week scan or evidence of sustained arrhythmia. Despite these restrictions, and the fact that high-risk patients without abnormality were not screened, the number of true-positive referrals continually increased over the project duration and exceeded the national average. Therefore, one may argue that opening fetal cardiac referrals to all of the recommended criteria ${ }^{4-8}$ may result in further improvement in the antenatal detection rate of heart anomalies. This prediction will be tested in the near future after full commissioning of the fetal cardiac service in Wales.

\section{Clinical governance and quality control measures}

Robust audit and quality control measures were central to establishing the efficacy of our work in this project. Openness of its results and public accountability are notable strengths of this study that antenatal cardiac anomaly detection rates and surgical outcomes of each heart defect in all specialist centres in the UK are available and accessible by the general public on CARIS and NICOR websites. ${ }^{34}$ Ongoing audit on a local and national level enabled identification of both good practice, shortcomings, patient experience and satisfaction. Addressing issues locally enabled them to be dealt with in a timely manner, which helped to minimise dissatisfaction in the patient experience, potential diagnostic errors, litigation, and a reduction in standards and in subsequent quality of care. The supportive teamworking ethos of the network enabled such feedback to be undertaken in a constructive way, with no fear of reprisal or undermining of clinical skills or knowledge. This was further enhanced by improvement in the communication networks, facilitated by regular meetings and study events. Further training opportunities on a one-to-one basis were offered for those staff requiring additional support, either self-declared or identified through audit.
The main message that we would like to convey to others A successful fetal cardiology service operating at each district general hospital in the UK is a realistic proposition. In fact, FASP in England used our achievement as an exemplar of best practice and started a similar teaching and training programme to improve standards in its district general hospitals around the country.

\section{Further recommendations}

The problems encountered during the process of developing learning activities and policy changes helped identify gaps in service provision and commissioning. These learning outcomes and experiences led to the successful preparation of an official business case for full commissioning of the fetal cardiac service for Wales in 2016.

\section{Limitations of the study}

This project was designed and delivered by enthusiastic individuals with dedication and hard work in a resource-limited environment. Therefore, it may not appear to project well the true financial implications of the proposed business model and its applicability in other settings. However, its validity was tested and it was shown to be effective in other screening programmes such as FASP in the UK.

\section{Reflection on the study}

Implementation of such a geographically extensive training programme requires careful coordination, planning and organisation. While the financial commitment can be minimised, in order for consistent and ongoing improvement in detection rates, engagement from all institutions and individual participants undertaking antenatal ultrasound screening is required. Trainer availability was often limited by clinical commitments and therefore for continued sustainability of an ongoing programme, as is required for maintenance of skills and knowledge, it is necessary to have a number of trainers. A greater number of trainers would enable greater preplanning of dates to ensure maximum staff availability for attendance as well as a more continuous and structured timing of training days. The subsequent development of cardiac leads to deliver ongoing training locally in each institution has addressed the issue of training availability and sustainability. On reflection, this could have been implemented much earlier in the process leading to greater efficiency in the time management of training sessions and reducing the time scale required for training the entire workforce.

Under the auspices of ASW and supported by a specialist fetal cardiologist, cardiac leads continue to develop their own personal skills to promote best practice and provide local support and training. As a result, sonographers strive to improve their skills to achieve higher detection rates of fetal cardiac abnormalities in Wales.

\section{CONCLUSIONS}

Mass training and skill dissemination programmes on the antenatal detection of CHD can be delivered with 
minimum impact on finite healthcare resources. Even though effective leadership and human factors are the main determinants for success, a safe and sustainable service can only be established by supporting the confidence and competency of the regional screening workforce through team building approaches, and lifelong learning activities. These activities must be reinforced by continuously updated policies and protocols in addition to robust clinical governance and audit practices.

\section{Author affiliations}

${ }^{1}$ Department of Paediatric Cardiology, University Hospital of Wales, Cardiff, UK

${ }^{2}$ School of Sport and Exercise Sciences and College of Engineering, Swansea University, Medical Physics and Biomedical Engineering, Swansea, UK

${ }^{3}$ School of Health Sciences, Cardiff University, Cardiff, UK

${ }^{4}$ Department of Radiology, Royal Glamorgan Hospital, Llantrisant, UK

${ }^{5}$ Department of Paediatrics, Princess of Wales Hospital, Bridgend, UK

${ }^{6}$ Department of Radiology, University Hospital of Wales, Cardiff, UK

${ }^{7}$ Department of Obstetric of Gynaecology, Nevill Hall Hospital, Abergavenny, UK

${ }^{8}$ Public Health Wales, Antenatal Screening Wales, Cardiff, UK

${ }^{9}$ Public Health Wales, Congenital Anomaly Register Information Service, Swansea, UK

Acknowledgements The authors thank all participants in the study and in particular, Welsh Fetal Cardiovascular Network; ultrasonographers undertaking fetal anomaly screening clinics in all Welsh hospitals; Public Health Wales; Welsh Health Specialist Services Commissioners; Antenatal Screening Wales; Tiny Tickers charity; fetal medicine and obstetric specialists, radiologists, paediatricians with a specialist interest in cardiology, superintendent sonographers and screening midwives at all Welsh hospitals; Cardiff and Vale University Health Board; parents, patients and charities supporting educational activities by giving talks or funding meetings; past and present research and visiting fellows.

Contributors OU conceived, designed and conducted the study; delivered teaching and training alongside ASW and Tiny Tickers; OU, NT and JK organised courses, workshops and educational conferences in collaboration with ASW and the Welsh Fetal Cardiovascular Network; OU, JK, CD, DT, DR, MJL, AT, NT, BB, AG analysed and interpreted the data, and drafted, finalised and submitted the manuscript. JK, CD, $\mathrm{DT}, \mathrm{DR}, \mathrm{MJL}, \mathrm{AT}, \mathrm{NT}, \mathrm{BB}, \mathrm{AG}, \mathrm{OU}$ interpreted the data and revised the manuscript for important intellectual content. AT, DR, AG, BB, OU, JK, NT, CD, DT and MJL had full access to all of the data in the study and can take responsibility for the integrity of the data and the accuracy of the data analysis. All authors approved the final version to be published.

Funding This paper had no external funding. Training programme continued until 2006 solely as individual effort of (OU, NT and screening midwives in South Wales) then Antenatal Screening Wales provided parallel training for sonographers through central funding from Public Health Wales. Additional event sponsorships were received from the exhibitors who attended annual conferences or workshops. Subsequent funding was awarded from Public Health Wales for a more structured training programme which was devised and delivered via Antenatal Screening Wales and Tiny Tickers Charity. Toshiba (Canon), General Electric, Philips, Siemens, and Samsung ultrasound divisions lent their application and equipment support for hands on courses. The funders had no role in the design and conduct of this paper; the collection, management, analysis and interpretation of the data and the preparation, review or approval of the manuscript.

Competing interests OU, NT, JK, AT and CD were all affiliated with Antenatal Screening Wales during defined periods to act as advisor on ultrasound experts advisory panel. OU and MJL are Professors at Swansea University. AT is an officer at ASW. OU and NT are appointed by ASW as responsible educators for delivering ongoing training to Cardiac Leads in each District General Hospital in Wales. DT is the CARIS manager. This study is part of the "Reducing perinatal mortality and improving postnatal outcomes from congenital heart disease by increasing antenatal anomaly detection programme" which won the BMJ Group Best Cardiology Team Award in the UK, in 2016.

Patient consent Not required.

Provenance and peer review Commissioned; externally peer reviewed.

Open access This is an open access article distributed in accordance with the Creative Commons Attribution Non Commercial (CC BY-NC 4.0) license, which permits others to distribute, remix, adapt, build upon this work non-commercially, and license their derivative works on different terms, provided the original work is properly cited, appropriate credit is given, any changes made indicated, and the use is non-commercial. See: http://creativecommons.org/licenses/by-nc/4.0/.

\section{REFERENCES}

1. Abu-Harb M, Wyllie J, Hey E, et al. Presentation of obstructive left heart malformations in infancy. Arch Dis Child Fetal Neonatal Ed 1994;71:F179-83.

2. All wales perinatal survey. http://medicine.cf.ac.uk

3. CARIS report on Congenital Heart Anomalies Swansea Wales. http:// www.wales.nhs.uk

4. NICOR. Congenital Cardiac Anomaly Database Portal. https://nicor4. nicor.org.uk/CHD/an_paeds.nsf/vwContent/Antenatal\%20Diagnosis? Opendocument

5. Gardiner HM. Antenatal detection of heart defects is important and achievable. Ultrasound 2005;13:164-9.

6. Royal College of Obstetrician Guidelines. http://www.rcog.org.uk/ womens-health/clinical-guidance/ultrasound-screening

7. BCCA Fetal Cardiology Standards. http://www.bcs.com/documents/ Fetal Cardiology Standards Final Version March 2010.pdf

8. ISUOG Guidelines. http://www.isuog.org/NR/rdonlyres/7C5CD9C4CC92-412E-A5A1-89FA77F59F81/0/ISUOGCardiacScreening.pdf

9. Gardiner HM. Fetal echocardiography: 20 years of progress. Heart 2001;86(Suppl 2):II12-22.

10. Carvalho JS, Mavrides E, Shinebourne EA, et al. Improving the effectiveness of routine prenatal screening for major congenital heart defects. Heart 2002;88:387-91.

11. Tegnander E, Eik-Nes SH. The examiner's ultrasound experience has a significant impact on the detection rate of congenital heart defects at the second-trimester fetal examination. Ultrasound Obstet Gynecol 2006;28:8-14.

12. Oggè G, Gaglioti P, Maccanti S, et al. Prenatal screening for congenital heart disease with four-chamber and outflow-tract views: a multicenter study. Ultrasound Obstet Gynecol 2006;28:779-84.

13. Galindo A, Herraiz I, Escribano D, et al. Prenatal detection of congenital heart defects: a survey on clinical practice in Spain. Fetal Diagn Ther 2011;29:287-95.

14. Randall P, Brealey S, Hahn S, et al. Accuracy of fetal echocardiography in the routine detection of congenital heart disease among unselected and low risk populations: a systematic review. BJOG 2005;112:24-30.

15. National Institute for Clinical Excellence guidelines for antenatal care. http://www.nice.org.uk/nicemedia/live/11947/40145/40145.pdf

16. Tworetzky W, McElhinney DB, Reddy VM, et al. Improved surgical outcome after fetal diagnosis of hypoplastic left heart syndrome. Circulation 2001;103:1269-73.

17. Sivarajan V, Penny DJ, Filan P, et al. Impact of antenatal diagnosis of hypoplastic left heart syndrome on the clinical presentation and surgical outcomes: the Australian experience. J Paediatr Child Health 2009;45:112-7.

18. Franklin O, Burch $\mathrm{M}$, Manning $\mathrm{N}$, et al. Prenatal diagnosis of coarctation of the aorta improves survival and reduces morbidity. Heart 2002;87:67-9.

19. Bonnet D, Coltri A, Butera G, et al. Detection of transposition of the great arteries in fetuses reduces neonatal morbidity and mortality. Circulation 1999;99:916-8.

20. Kumar RK, Newburger JW, Gauvreau K, et al. Comparison of outcome when hypoplastic left heart syndrome and transposition of the great arteries are diagnosed prenatally versus when diagnosis of these two conditions is made only postnatally. Am J Cardiol 1999;83:1649-53. 